\title{
Analyst
}

\section{Enhancing permanganate chemiluminescence detection for the determination of glutathione and glutathione disulfide in biological matrices $\dagger$}

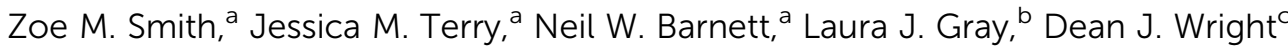 \\ and Paul S. Francis*a
}

Acidic potassium permanganate chemiluminescence enables direct post-column detection of glutathione, but its application to assess the redox state of a wider range of biological fluids and tissues is limited by its sensitivity. Herein we show that the simple on-line addition of an aqueous formaldehyde solution not only enhances the sensitivity of the procedure by two orders of magnitude, but also provides a remarkable improvement in the selectivity of the reagent towards thiols such as glutathione (compared to phenols and amino acids that do not possess a thiol group). This enhanced mode of detection was applied to the determination of glutathione and its corresponding disulfide species in homogenised striatum samples taken from both wild type mice and the R6/1 transgenic mouse model of Huntington's disease, at both 8 and 12 weeks of age. No significant difference was observed between the GSH/GSSG ratios of wild type mice and R6/1 mice at either age group, suggesting that the early disease progression had not significantly altered the intracellular redox environment.

Received 5th February 2014

Accepted 24th March 2014

DOI: 10.1039/c4an00255e

www.rsc.org/analyst derivatisation), such as electrochemical ${ }^{9}$ and chemiluminescence, ${ }^{\mathbf{1 0 - 1 2}}$ are therefore an attractive alternative to fluorescence. ${ }^{6,7,13,14}$

Several new approaches for chemiluminescence detection of GSH have recently emerged. ${ }^{\mathbf{1 0 - 1 2 , 1 5}} \mathrm{Li}$ et al. reported the determination of thiols such as cysteine, GSH, $N$-acetylcysteine and captopril based on the sensitised chemiluminescence reaction with acidic potassium permanganate and quinine. ${ }^{15}$ This system actually contains two light producing pathways: (i) the reduction of permanganate to form an excited state manganese(II) species, and (ii) the oxidation of the thiols to generate an excited intermediate capable of transferring energy to the quinine fluorophore. ${ }^{\mathbf{1 6}}$ The manganese(II) pathway can be considerably enhanced by addition of sodium polyphosphate rather than quinine, which improves sensitivity by eliminating the background emission resulting from the reaction between the sensitiser and oxidant. ${ }^{17,18}$ In 2011, McDermott et al. utilised this approach for the post-column chemiluminescence detection of GSH in cultured skeletal muscle cells (C2C12 myotubes) following a rapid isocratic separation. ${ }^{11}$ The limit of detection was $5 \times 10^{-7} \mathrm{M}$, compared to $6.5 \times 10^{-6} \mathrm{M}$ reported by Li and co-workers. ${ }^{11,15}$ McDermott and co-workers also utilised a colloidal manganese(Iv) reagent for the direct post-column chemiluminescence detection of thiols and disulfides. The limits of detection of $7 \times 10^{-8} \mathrm{M}$ for GSH and $1 \times 10^{-7} \mathrm{M}$ for GSSG were suitable for their determination in whole blood samples, ${ }^{12}$ but they are inadequate to measure the relatively low concentration of GSSG in various other clinically relevant physiological fluids, cells and tissues. 
Herein we investigate a range of enhancers in order to improve the sensitivity of acidic potassium permanganate chemiluminescence reagent towards thiol compounds. We show that the on-line addition of an aqueous formaldehyde solution can not only improve the sensitivity towards thiol compounds, but also dramatically enhance the selectivity of the reagent. We apply this enhanced chemiluminescence reagent system to the determination of GSH and GSSG in mouse striatum.

\section{Experimental}

\section{Chemicals}

Deionised water (Continental Water Systems, Victoria, Australia) and analytical grade reagents were used unless otherwise stated. Chemicals were obtained from the following sources: $N$ acetylcysteine (Nacys), $\mathrm{N}$-cyclohexyl-3-aminopropanesulfonic acid (CAPS), L-cysteine (Cys), L-cystine (CYSS), $N$-ethylmaleimide (NEM), L-glutathione (GSH), L-glutathione disulfide (GSSG), glyoxal, homocysteine (Hcys), homocystine (HCYSS), L-lysine (Lys), 2-mercaptoethanol, L-methionine (Met), L-phenylalanine (Phe), quinine, serotonin hydrochloride (5-HT), sodium polyphosphate, tris(2-carboxyethyl)phosphine hydrochloride (TCEP), L-tryptophan (Trp) and L-tyrosine (Tyr) from Sigma-Aldrich (New South Wales, Australia); potassium permanganate, and formaldehyde (37\%) from Chem-Supply (South Australia, Australia); methanol, sulfuric acid, and tris(hydroxymethyl)methylamine from Merck (Victoria, Australia); morphine from GlaxoSmithKline (Victoria, Australia), formic acid from Hopkin and Williams (Essex, England) and hydrochloric acid (32\% w/v) from Ajax Finechem (New South Wales, Australia).

Stock solutions $\left(1 \times 10^{-3} \mathrm{M}\right)$ of $N$-acetylcysteine, cysteine, cystine, GSH, GSSG, homocysteine and homocystine were prepared and diluted as required in $0.01 \%$ formic acid ( $\mathrm{pH} 2.8$ ). Formaldehyde was filtered and diluted to the required concentration with deionised water. The acidic potassium permanganate reagent $\left(2.5 \times 10^{-4} \mathrm{M}\right)$ was prepared by dissolving solid potassium permanganate in a solution of sodium polyphosphate $(1 \%(\mathrm{~m} / \mathrm{v}))$ and adjusting to $\mathrm{pH} 3$ with sulfuric acid.

\section{Flow injection analysis}

The manifold was constructed from a Gilson Minipuls 3 peristaltic pump (John Morris Scientific, NSW, Australia) with bridged PVC pump tubing (white/white, $1.02 \mathrm{~mm}$ i.d., DKSH, Queensland, Australia), PTFE manifold tubing $(0.8 \mathrm{~mm}$ i.d., Cole-Parmer Instrument Company, Illinois, USA) and a six-port injection valve (Vici 04W-0192L Valco Instruments, Texas, USA) equipped with a $70 \mu \mathrm{L}$ sample loop. A custom built flow-cell (a tight coil of $0.8 \mathrm{~mm}$ i.d. PTFE tubing) was mounted against an extended range photomultiplier tube (Electron Tubes model 9828SB, ETP, NSW, Australia) and encased in a light-tight housing. The output signal from the detector was obtained using an e-corder 410 data acquisition system (eDAQ, NSW, Australia). Analytes were injected into a carrier stream (deionised water) which merged with a stream of either deionised water or formaldehyde ( $2 \mathrm{M}$ ) at a confluence point located at the entrance of a $1 \mathrm{~m}$ reaction coil. This stream was then combined with the acidic potassium permanganate reagent just prior to entry into the flow-cell.

\section{High performance liquid chromatography}

Chromatographic analysis was carried out on an Agilent Technologies 1260 series liquid chromatography system, equipped with a quaternary pump, solvent degasser system, and autosampler (Agilent Technologies, Forest Hill, Victoria, Australia) using an Alltech Alltima C18 column $(250 \mathrm{~mm} \times 4.6 \mathrm{~mm}$ i.d., 5 $\mu \mathrm{m})$ equipped with an Alltima C18 guard column, at room temperature, with an injection volume of $10 \mu \mathrm{L}$ and a flow rate of $1 \mathrm{~mL} \mathrm{~min}{ }^{-1}$. Isocratic elution was performed with $97 \%$ solvent A (deionised water adjusted to $\mathrm{pH} 2.8$ with formic acid) and $3 \%$ solvent $\mathrm{B}$ (methanol). An analogue to digital interface box (Agilent Technologies) was used to convert the signal from the chemiluminescence detector. Before use in the HPLC system, all sample solutions and solvents were filtered through a $0.45 \mu \mathrm{m}$ nylon membrane. For post-column chemiluminescence measurements the column eluate $\left(1 \mathrm{~mL} \mathrm{~min}{ }^{-1}\right)$ and a formaldehyde enhancer $\left(2 \mathrm{M} ; 1 \mathrm{~mL} \mathrm{~min}^{-1}\right)$ were merged at a T-piece located $10 \mathrm{~cm}$ from the entrance of the detector. This stream was then combined with the potassium perman-

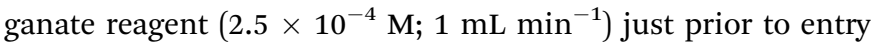
into the flow-cell. A GloCel chemiluminescence detector (Global FIA, WA, USA) containing a dual-inlet serpentine flow-cell and photomultiplier tube (model 9828SB, ETP, NSW, Australia) set at a constant voltage of $900 \mathrm{~V}$ from a stable power supply (PM20D, ETP) was utilised in this system. The reagent and enhancer solutions were propelled by a $12 \times 6$ Dual Piston Pump (Scientific Systems Inc., PA, USA) equipped with a pulse damper and self-flushing pump heads.

\section{Chemiluminescence spectra}

A Cary Eclipse fluorescence spectrophotometer (Varian, Mulgrave, Victoria, Australia) equipped with R928 photomultiplier tube (Hamamatsu, Japan) was operated in bio/chemiluminescence mode to collect chemiluminescence spectra. A two-line flow manifold was used to continuously merge the analyte $\left(5 \times 10^{-5} \mathrm{M} ; 3.5 \mathrm{~mL} \mathrm{~min}^{-1}\right)$ and reagent solutions $(2.5 \times$ $10^{-4} \mathrm{M} ; 3.5 \mathrm{~mL} \mathrm{~min}^{-1}$ ) at a confluence point located just prior to the entrance of a coiled PTFE flow-cell $(200 \mu \mathrm{L}, 0.8 \mathrm{~mm}$ i.d.) that was mounted against the emission window of the spectrophotometer. A three-line continuous flow manifold was subsequently used to merge the analyte solution $\left(1 \times 10^{-7} \mathrm{M}\right.$; $\left.3.5 \mathrm{~mL} \mathrm{~min}^{-1}\right)$ with the formaldehyde enhancer $(2.0 \mathrm{M} ; 3.5 \mathrm{~mL}$ $\mathrm{min}^{-1}$ ) at a confluence point located at the entrance of a short $(1 \mathrm{~m})$ reaction coil. This stream was then combined with the acidic potassium permanganate reagent $\left(2.5 \times 10^{-4} \mathrm{M} ; 3.5 \mathrm{~mL}\right.$ $\min ^{-1}$ ) just prior to entry into the flow-cell. Final spectra were an average of 20 scans (1 s gate time; $1 \mathrm{~nm}$ data interval; $20 \mathrm{~nm}$ band-pass; PMT, $800 \mathrm{~V}$ ) and corrected as previously described. ${ }^{\mathbf{9}}$

\section{Mice}

R6/1 transgenic hemizygote males ${ }^{\mathbf{2 0}}$ were originally obtained from the Jackson Laboratory (Bar Harbor, ME, USA) and bred 
with CBB6 (CBAA $\times$ C57/B6) F1 females to establish the R6/1 colony at the Florey Institute of Neuroscience and Mental Health (FINMH). After weaning, animals were group housed (4 mice per cage with 2 of each genotype) and maintained on a 12 $\mathrm{h}$ light/dark cycle with access to food and water ad libitum. Mice were handled in accordance with the guidelines of the FINMH Animal Ethics Committee and the National Health and Medical Research Council (NHMRC).

\section{Sample collection}

Immediately after cervical dislocation, mice brains were dissected on ice and snap frozen in liquid nitrogen, before being stored at $-80{ }^{\circ} \mathrm{C}$. Tissue was allowed to defrost on ice prior to homogenization. Tissue homogenates were prepared in $0.1 \%$ formic acid, using a motorized pestle. Samples were allowed to rest on ice for $10 \mathrm{~min}$ prior to centrifugation at 8000 $\mathrm{G}$ for $15 \mathrm{~min}$. Supernatants were removed for analysis.

\section{Sample analysis}

For GSH determination the sample was initially diluted 10 -fold into aqueous formic acid (0.01\%). A $50 \mu \mathrm{L}$ aliquot of the diluted supernatant was then combined with $450 \mu \mathrm{L}$ of aqueous formic acid (5\%), immediately prior to analysis using HPLC with acidic potassium permanganate chemiluminescence detection. For GSSG determination, a second aliquot of the diluted supernatant $(100 \mu \mathrm{L})$ was combined with a Tris-HCl buffer $(0.675 \mathrm{M} ; 20$ $\mu \mathrm{L} ; \mathrm{pH} 8.0)$ and NEM $\left(6.3 \times 10^{-3} \mathrm{M} ; 20 \mu \mathrm{L}\right)$ and mixed for $30 \mathrm{~s}$. 2-Mercaptoethanol $\left(8 \times 10^{-3} \mathrm{M} ; 20 \mu \mathrm{L}\right)$ was then added and mixed for a further $30 \mathrm{~s}$. Following addition of TCEP $\left(7.8 \times 10^{-4}\right.$ $\mathrm{M} ; 20 \mu \mathrm{L}$ ) the solution was gently heated at $50{ }^{\circ} \mathrm{C}$ for $60 \mathrm{~min}$ to allow complete disulfide bond reduction. Finally, aqueous formic acid $(5 \%, 20 \mu \mathrm{L})$ was introduced to re-acidify the sample prior to filtration and analysis.

\section{Results and discussion}

\section{Preliminary investigations}

In an attempt to provide a more analytically useful signal from $\mathrm{GSH}$, four compounds previously reported to enhance the emission intensity from the reaction of various organic compounds with acidic potassium permanganate ${ }^{17,18,21-23}$ were examined: $N$-cyclohexyl-3-aminopropanesulfonic acid (CAPS), quinine, glyoxal and formaldehyde. Using a three-line flow injection analysis manifold, a GSH solution $\left(1 \times 10^{-6} \mathrm{M}\right)$ was injected into a deionised water carrier $\left(3.5 \mathrm{~mL} \mathrm{~min}^{-1}\right)$ which merged with the enhancer solution $\left(3.5 \mathrm{~mL} \mathrm{~min}^{-1}\right)$ at a confluence point located at the entrance of a short reaction coil. A continuously flowing stream of the reagent $\left(2.5 \times 10^{-4} \mathrm{M} ; 3.5\right.$ $\mathrm{mL} \min ^{-1}$ ) then merged with the combined analyte-enhancer solution just prior to entry into the flow-cell. Under these conditions, each of the enhancers resulted in emissions greater than that obtained with only deionised water in the enhancer stream. A small improvement in the chemiluminescence signal was obtained when using solutions of CAPS, quinine and glyoxal at $1.0 \mathrm{mM}$ (Fig. 1). A much larger increase in signal intensity was observed with a more concentrated solution of glyoxal ( $\sim 600$-fold enhancement), but this response was lower than that obtained from the corresponding blank (i.e. the reaction of permanganate and the enhancer without GSH). The addition of formaldehyde resulted in a significant increase in signal intensity (over three orders of magnitude), with a relatively small increase in the corresponding blank response.

\section{Confirmation of the emitting species}

The mechanism of enhancement of permanganate chemiluminescence systems by formaldehyde has not yet been elucidated. ${ }^{17}$ It has been postulated that the enhancer is oxidised to form excited singlet oxygen or an unknown intermediate that either emits light or transfers energy to other compounds. ${ }^{24-27}$

For the chemiluminescence reaction of GSH with the acidic potassium permanganate reagent, we observed a broad single band with an apparent emission maximum at approximately $687 \mathrm{~nm}$ (Fig. S1a $\dagger$ ), which is characteristic of the electronically excited manganese(II) species formed in the oxidation of organic analytes by permanganate in the presence of sodium polyphosphate $\left(\lambda_{\max }=689 \pm 5 \mathrm{~nm} ;{ }^{4} \mathrm{~T}_{1} \rightarrow{ }^{6} \mathrm{~A}_{1}\right.$ transition $\left.{ }^{28,29}\right)$. The incorporation of a formaldehyde enhancer ( $2 \mathrm{M}$ ) into the system, increased the overall emission intensity but did not alter the spectral distribution (Fig. S1b $\dagger$ ). It was therefore concluded that the same Mn(II) species was responsible for the emission in both cases.

\section{Analyte screen}

Fifteen analytes $\left(5 \times 10^{-6} \mathrm{M}\right)$ were screened with the acidic potassium permanganate reagent under reaction conditions previously found to afford the greatest chemiluminescence response for GSH. ${ }^{11}$ These responses (Fig. 2a) were then

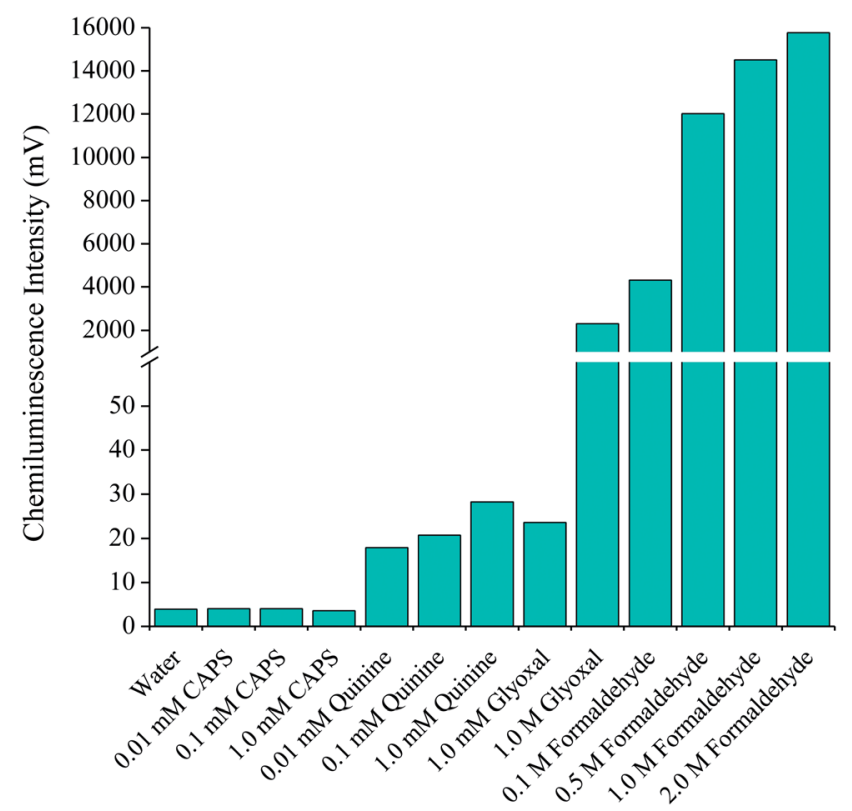

Fig. 1 Chemiluminescence intensities for GSH $\left(1 \times 10^{-6} \mathrm{M}\right)$ upon reaction with acidic potassium permanganate and various enhancer solutions using flow injection analysis. 
compared to those obtained when incorporating the formaldehyde enhancer (Fig. 2b). With the exception of glycine and lysine, the addition of formaldehyde increased the chemiluminescence intensity for each analyte. Moderate enhancement (59- to 197-fold) was obtained for species containing a disulfide bond (CYSS, HCYSS, GSSG). However, this improvement in sensitivity was still insufficient to allow direct postcolumn quantification of GSSG in clinical samples. The greatest improvements in signal intensity were obtained for the thiol containing species: Cys, Hcys, GSH and Nacys, with increases of between 528- and 4417-fold. Conversely, the response from two phenolic analytes that are known to produce very intense signals with the acidic potassium permanganate (morphine and serotonin) were only enhanced 2- to 3-fold. Therefore, the formaldehyde enhancer not only affords much greater sensitivity, but also provided a remarkable improvement in selectivity towards thiol compounds such as GSH, thus reducing potential interferences in complex biological samples.
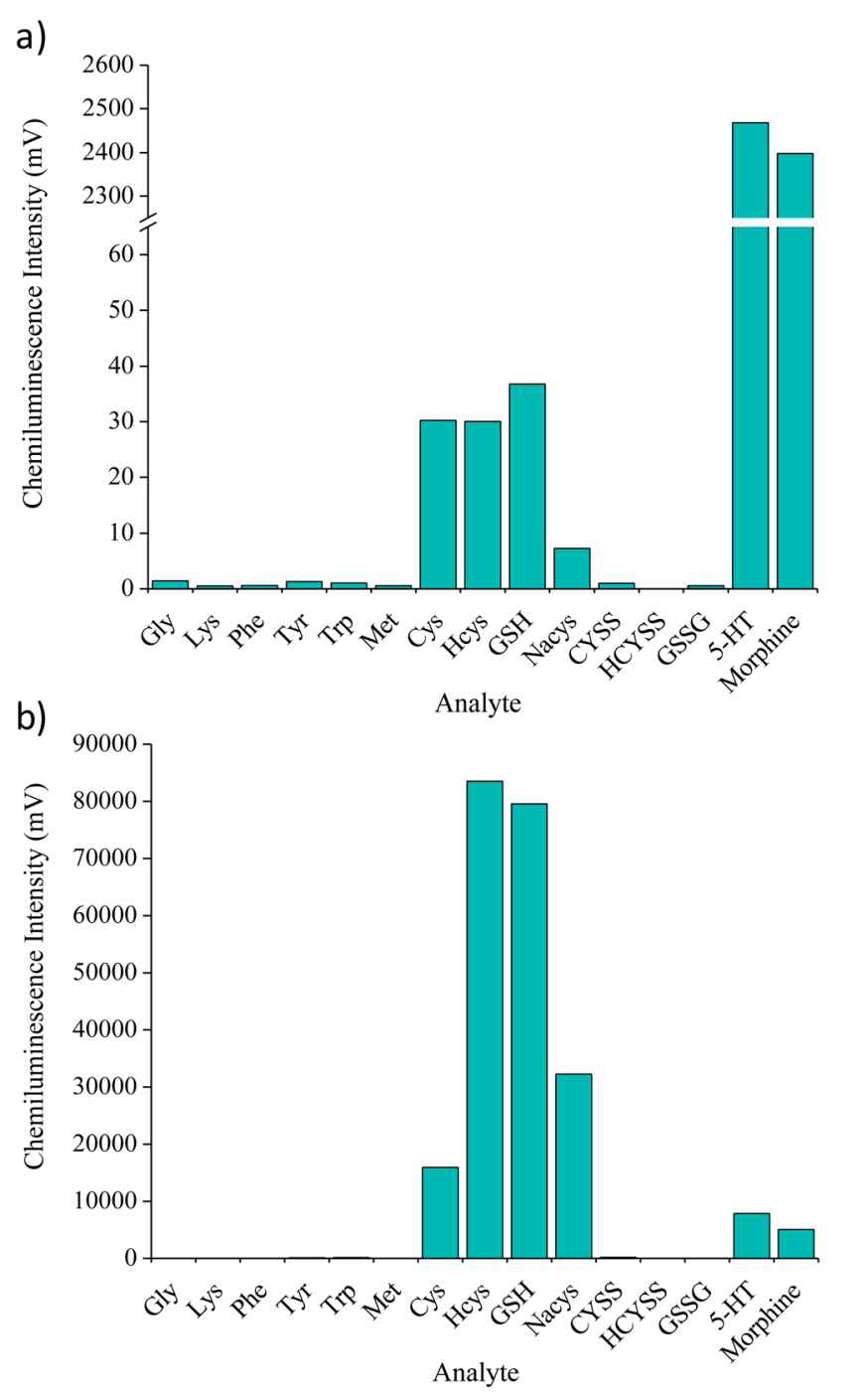

Fig. 2 Chemiluminescence intensities for selected analytes $\left(5 \times 10^{-6}\right.$ $\mathrm{M})$ with (a) acidic potassium permanganate $\left(2.5 \times 10^{-4} \mathrm{M}\right.$ in $1 \%(\mathrm{~m} / \mathrm{v})$ sodium polyphosphate, $\mathrm{pH} 3$ ) and (b) the permanganate reagent and formaldehyde, using flow injection analysis methodology.

\section{High performance liquid chromatography}

A mobile phase consisting of $97 \%$ deionised water adjusted to pH 2.8 with formic acid and 3\% methanol enabled the separation of four routinely measured thiols in under 15 min with high resolution (Fig. 3). Similar to the observations of the preliminary flow injection analysis experiments, the addition of $2 \mathrm{M}$ formaldehyde to the detection system resulted in an increase in the chemiluminescence response (peak area) for these thiol analytes by approximately two orders of magnitude.

It should be noted that signal increases obtained when using a formaldehyde enhancer are typically accompanied by a deleterious increase in background emission. ${ }^{\mathbf{1 7 , 1 8}}$ Peristaltic pumps (which are often used to propel reagent and enhancer solutions into the flow-cell for post-column chemiluminescence detection) contribute greatly to baseline noise because of the pulsation of solution flow. To minimise this effect, we employed two dual piston pumps equipped with pulse dampers to deliver reagent and enhancer streams to the detector.

Calibration curves were prepared using eighteen standard solutions between $1 \times 10^{-9} \mathrm{M}$ and $1 \times 10^{-5} \mathrm{M}$. Each of the thiols exhibited a highly linear relationship between signal (peak area) and analyte concentration (Table 1). The precision of repeated injections ( $n=10 ; 5 \times 10^{-6} \mathrm{M}$ ) was excellent, with relative standard deviations of between $0.49 \%$ and $1.46 \%$. Limits of detection, defined as a signal to noise ratio of 3 , were in the range of $1 \times 10^{-8} \mathrm{M}$ and $5 \times 10^{-8} \mathrm{M}$. Most importantly, the limit of detection for GSH was 50 -fold superior to that of the previously published HPLC procedure utilising permanganate chemiluminescence detection. ${ }^{\mathbf{1 1}}$

After quantification of the free GSH (Fig. 4a), a second aliquot of sample was used to establish the concentration of

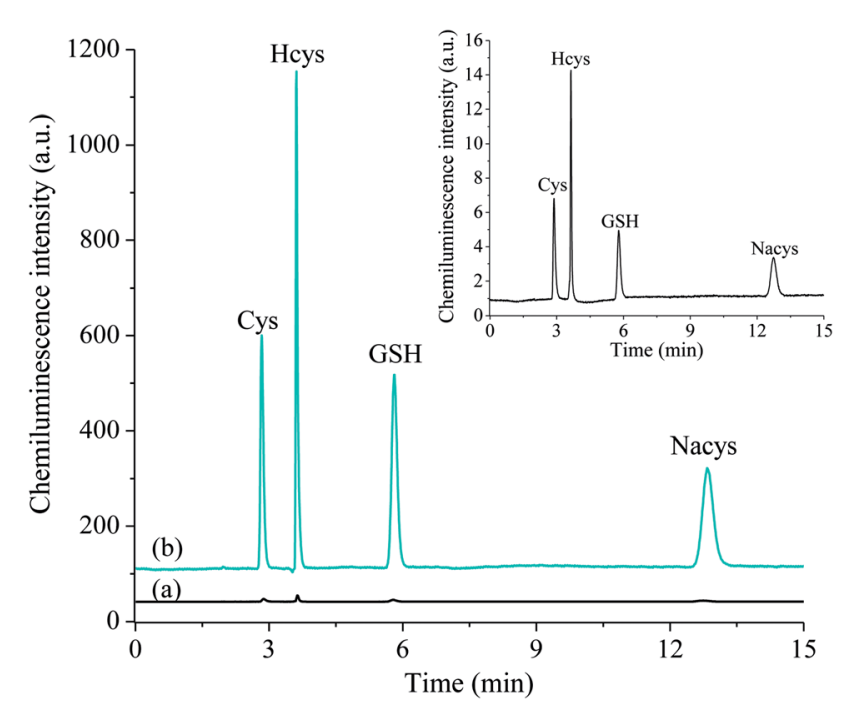

Fig. 3 HPLC separation of a mixture of four biologically important thiols; cysteine (Cys), homocysteine (Hcys), glutathione (GSH) and $N$ acetylcysteine (Nacys) $\left(1 \times 10^{-5} \mathrm{M}\right)$, using (a) acidic potassium permanganate $\left(2.5 \times 10^{-4} \mathrm{M}\right)$ chemiluminescence detection $(20 \mu \mathrm{L}$ injection volume) and (b) acidic potassium permanganate and formaldehyde $(2.0 \mathrm{M})(10 \mu \mathrm{L}$ injection). The unenhanced separation is also shown inset. 
Table 1 Analytical figures of merit

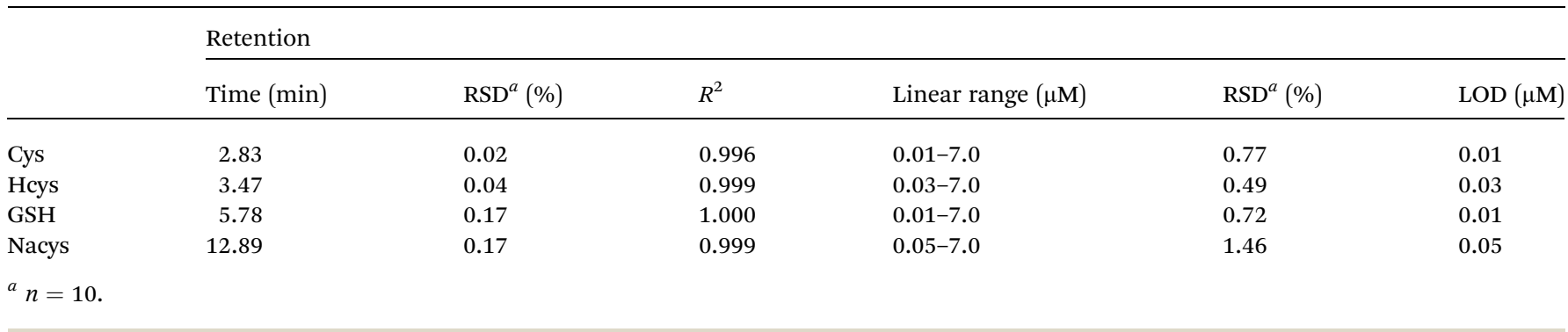

GSSG. The free thiol was blocked with the masking agent, $N$ ethylmaleimide (Fig. 4b) and then the disulfide bond reduction procedure was performed to liberate GSH, which was quantified as described above (Fig. 4c). As such, the gains in sensitivity for GSH were also translated to the detection of GSSG.

\section{Determination of GSH/GSSG in mouse striatum}

To demonstrate the viability of this method for the analysis of biological tissues, the procedure was applied to the determination of GSH and GSSG in mouse striatum. Progressive degeneration of this brain region is the primary neuropathalogic feature of Huntington's disease. ${ }^{\mathbf{3 0 , 3 1}}$ Several studies have reported signs of oxidative stress in the brain tissue of subjects suffering from the late stages of the disease; however, it is not clear if this begins in early stages or if it is secondary to tissue degeneration. ${ }^{31-33}$ Quantification of GSH and GSSG in animal models of Huntington's disease is therefore invaluable in determining the stage at which oxidative stress begins to occur.

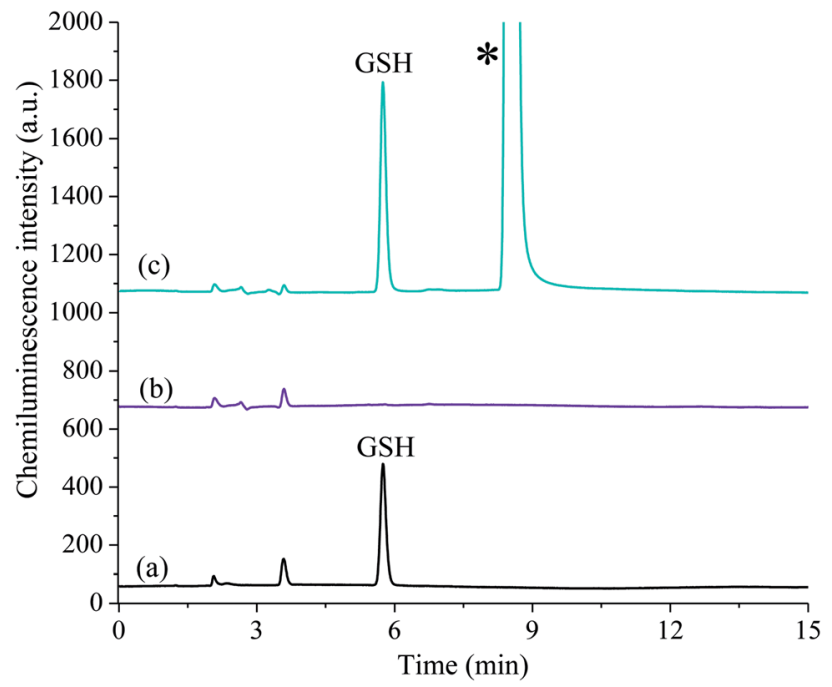

Fig. 4 The analysis of a mixture of GSH and GSSG standards $\left(1 \times 10^{-5}\right.$ M) with post-column chemiluminescence detection using acidic potassium permanganate and a formaldehyde enhancer. (a) Detection of $\mathrm{GSH}$, (b) signal after addition of Tris- $\mathrm{HCl}$ buffer and NEM (thiol blocking step), (c) detection of GSSG as GSH after the addition of 2mercaptoethanol (to react excess NEM) and TCEP (to break the disulfide bond). *This peak corresponds to the excess 2-mercaptoethanol and does not interfere with the analysis.
We examined homogenised striatum samples taken from both wild type mice and the R6/1 transgenic mouse model of Huntington's disease, at both 8 weeks of age (when affectivelike phenotypes begin to emerge ${ }^{34}$ ) and 12 weeks of age (when motor dysfunction occurs $\left.{ }^{20}\right)$. The acidic supernatant was initially diluted 10 -fold into aqueous formic acid $(0.01 \%)$. A portion of the diluted supernatant was then further diluted 10 -fold in 5\% formic acid immediately prior to analysis (Fig. 5a). For GSSG determination, a second aliquot of dilute supernatant $(100 \mu \mathrm{L})$ was subjected to the thiol blocking and disulfide bond reduction procedure as described above. The sample was then re-acidified with $20 \mu \mathrm{L}$ of $5 \%$ formic acid prior to analysis (Fig. 5b).

Using this method, both GSH and GSSG were detected in all samples tested. Despite the complexity of the sample matrix, the selectivity of the reagent combined with the ability to perform large dilutions resulted in only a few observable peaks from other compounds. GSH was present in the range 1.21$3.21 \mu \mathrm{mol} \mathrm{g}{ }^{-1}$ whilst GSSG was present in the range 0.16$0.44 \mu \mathrm{mol} \mathrm{g}^{-1}$ (Fig. 6). There was no significant difference in the levels of GSH, GSSG, or the ratio of GSH/GSSG, at either time

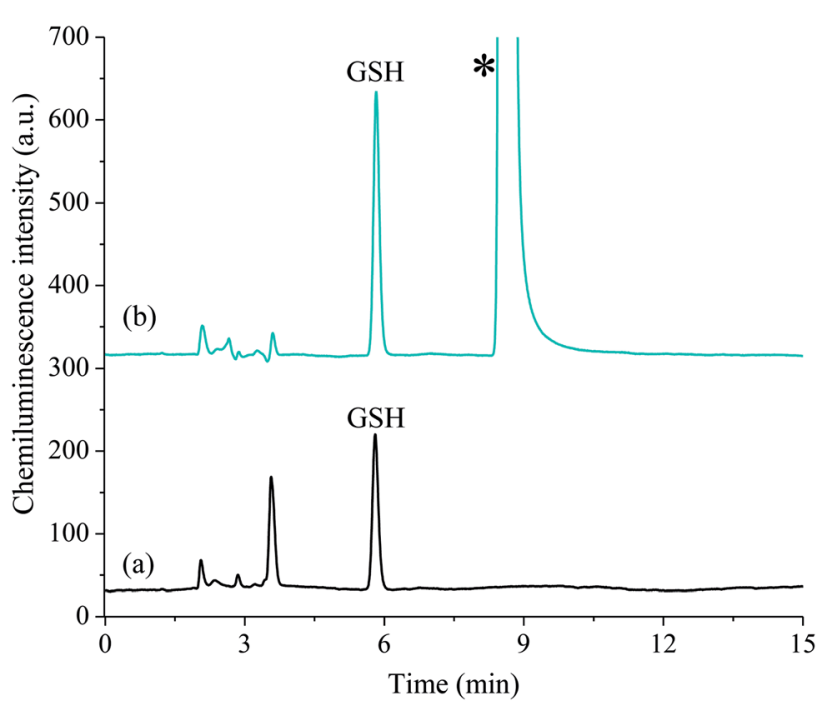

Fig. 5 Typical acidic potassium permanganate chemiluminescence chromatograms obtained for the analysis of mouse striatum, (a) detection of GSH (total 1/100 dilution) and (b) detection of GSSG as GSH after performing the disulfide bond reduction procedure (total $1 / 20$ dilution). *This peak corresponds to the excess 2 -mercaptoethanol and does not interfere with the analysis. 

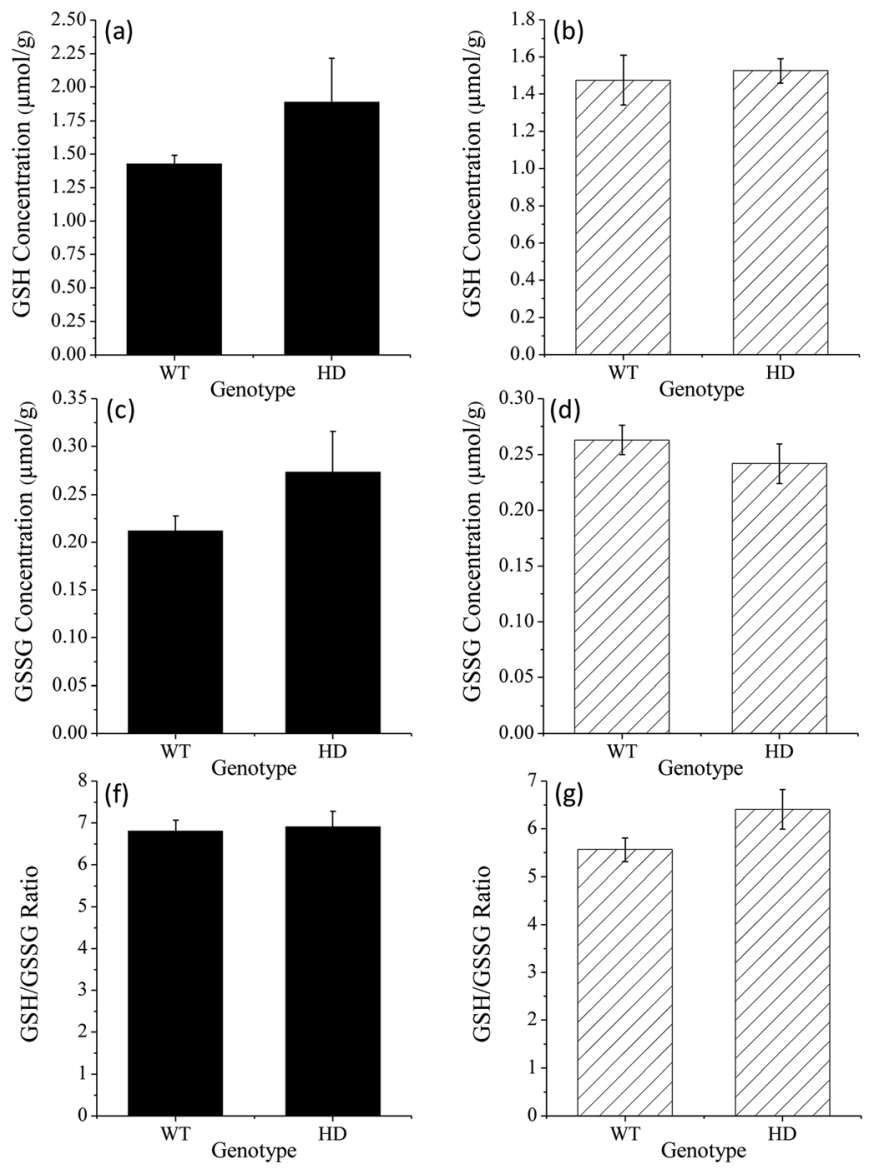

Fig. 6 Influence of genotype (wild type (WT) or R6/1 (HD)) on intracellular GSH, GSSG and the GSH/GSSG ratio in moue striatum taken from ( $a, c$, and e) 8 week old and (b, d, and f) 12 week old animals. Values are reported as means of 5 determinations $( \pm$ SEM).

point $(t(8)=2.89, p=0.20$; see Table $\mathrm{S} 1 \uparrow$ for further statistical data). This suggests that the disease progression had not significantly altered the intracellular redox environment. This preliminary result may indicate that significant changes in antioxidant capacity are not occurring in the prodromal stages of the R6/1 Huntington's disease model. However, it may be that such changes in glutathione homeostasis are compartmentalised between different cell types (glia and neurons) and/or subcellular structures (mitochondria and cytoplasm). Thus further analysis is needed to confirm if such changes are occurring. These results do, however, demonstrate the analytical utility of this method for the measurement of GSH/GSSG levels in complex biological matrices.

\section{Conclusions}

Applying a formaldehyde enhancer to chemiluminescence reactions with acidic potassium permanganate not only afforded significant increases in signal intensities, but also increased selectivity of the reagent towards thiol compounds. Applying this to the previously developed HPLC method for thiol detection, we were able to improve the detection limits for the important biological thiol GSH by over an order of magnitude whilst also halving the injection volume. The improved method was successfully applied to the detection of GSH/GSSG in mouse striatum.

\section{Acknowledgements}

The authors thank Deakin University and the Australian Research Council (FT100100646) for funding. The R6/1 mice were generously provided by Dr Anthony Hannan and Dr Thibault Renoir from the Florey Institute of Neuroscience and Mental Health, The University of Melbourne. The R6/1 colony was supported in part by a Future Fellowship (FT100100835) awarded to Dr Hannan. Dean Wright was supported by an Australian Postgraduate Award through the University of Melbourne.

\section{Notes and references}

1 A. Meister and M. E. Anderson, Annu. Rev. Biochem., 1983, 52, 711-760.

2 D. Giustarini, I. Dalle-Donne, D. Tsikas and R. Rossi, Crit. Rev. Clin. Lab. Sci., 2009, 46, 241-281.

3 A. Pastore, G. Federici, E. Bertini and F. Piemonte, Clin. Chim. Acta, 2003, 333, 19-39.

4 D. M. Townsend, K. D. Tew and H. Tapiero, Biomed. Pharmacother., 2003, 57, 145-155.

5 M. Valko, D. Leibfritz, J. Moncol, M. T. D. Cronin, M. Mazur and J. Telser, Int. J. Biochem. Cell Biol., 2007, 39, 44-84.

6 T. Toyo'oka, J. Chromatogr. B, 2009, 877, 3318-3330.

7 P. Monostori, G. Wittmann, E. Karg and S. Túri, J. Chromatogr. B, 2009, 877, 3331-3346.

8 R. E. Hansen and J. R. Winther, Anal. Biochem., 2009, 394, 147-158.

9 J. C. Harfield, C. Batchelor-McAuley and R. G. Compton, Analyst, 2012, 137, 2285-2296.

10 S. Bai, Q. Chen, C. Lu and J.-M. Lin, Anal. Chim. Acta, 2013, 768, 96-101.

11 G. P. McDermott, P. S. Francis, K. J. Holt, K. L. Scott, S. D. Martin, N. Stupka, N. W. Barnett and X. A. Conlan, Analyst, 2011, 136, 2578-2585.

12 G. P. McDermott, J. M. Terry, X. A. Conlan, N. W. Barnett and P. S. Francis, Anal. Chem., 2011, 83, 6034-6039.

13 E. Camera and M. Picardo, J. Chromatogr. B, 2002, 781, 181206.

14 F. Carlucci and A. Tabucchi, J. Chromatogr. B, 2009, 877, 3347-3357.

15 Y. Li, A. Zhang, J. Du and J. Lu, Anal. Lett., 2003, 36, 871-879.

16 J. L. Adcock, P. S. Francis and N. W. Barnett, J. Fluoresc., 2009, 19, 867-874.

17 J. L. Adcock, P. S. Francis and N. W. Barnett, Anal. Chim. Acta, 2007, 601, 36-67.

18 J. L. Adcock, N. W. Barnett, C. J. Barrow and P. S. Francis, Anal. Chim. Acta, 2014, 807, 9-28.

19 P. S. Francis, J. L. Adcock and N. W. Barnett, Spectrochim. Acta, Part A, 2006, 65, 708-710.

20 L. Mangiarini, K. Sathasivam, M. Seller, B. Cozens, A. Harper, C. Hetherington, M. Lawton, Y. Trottier, H. Lehrach, et al., Cell, 1996, 87, 493-506. 
21 N. T. Deftereos, A. C. Calokerinos and C. E. Efstathiou, Analyst, 1993, 118, 627-632.

22 J. A. Murillo Pulgarín, P. Fernández López and P. Hoyas Nuño, Anal. Bioanal. Chem., 2006, 384, 423-430.

23 J. A. Murillo Pulgarín, L. F. García Bermejo and P. Fernández López, Anal. Chim. Acta, 2005, 546, 60-67.

24 G. N. Chen, F. X. Huang, X. P. Wu, Z. F. Zhao and J. P. Duan, Anal. Bioanal. Chem., 2003, 376, 873-878.

25 S. Liao, X. Wu and Z. Xie, Anal. Chim. Acta, 2005, 537, 189195.

26 S. Fan, Z. Wu, L. Zhang and C. Lu, Anal. Lett., 2002, 35, 14791489.

27 H.-Q. Wei and E.-B. Liu, J. Chin. Chem. Soc., 2005, 52, 10431048.

28 N. W. Barnett, B. J. Hindson, P. Jones and T. A. Smith, Anal. Chim. Acta, 2002, 451, 181-188.
29 J. L. Adcock, P. S. Francis, T. A. Smith and N. W. Barnett, Analyst, 2008, 133, 49-51.

30 J. P. Vonsattel, R. H. Myers, T. J. Stevens, R. J. Ferrante, E. D. Bird and E. P. Richardson Jr, J. Neuropathol. Exp. Neurol., 1985, 44, 559-577.

31 S. E. Browne, A. C. Bowling, U. MacGarvey, M. J. Baik, S. C. Berger, M. M. K. Muqit, E. D. Bird and M. F. Beal, Ann. Neurol., 1997, 41, 646-653.

32 S. E. Browne, R. J. Ferrante and M. F. Beal, Brain Pathol., 1999, 9, 147-163.

33 M. A. Sorolla, G. Reverter-Branchat, J. Tamarit, I. Ferrer, J. Ros and E. Cabiscol, Free Radical Biol. Med., 2008, 45, 667-678.

34 T. Renoir, M. S. Zajac, X. Du, T. Y. Pang, L. Leang, C. Chevarin, L. Lanfumey and A. J. Hannan, PLoS One, 2011, 6, e22133. 